\title{
B cell activator PAX5 promotes lymphomagenesis through stimulation of $B$ cell receptor signaling
}

\author{
Diana Cozma, ${ }^{1}$ Duonan Yu, ${ }^{1}$ Suchita Hodawadekar, ${ }^{2}$ Anna Azvolinsky, ${ }^{1}$ Shannon Grande, ${ }^{3}$ \\ John W. Tobias, ${ }^{4}$ Michele H. Metzgar, ${ }^{5}$ Jennifer Paterson, ${ }^{6}$ Jan Erikson, ${ }^{5}$ Teresa Marafioti, ${ }^{6}$ \\ John G. Monroe, ${ }^{3}$ Michael L. Atchison, ${ }^{2}$ and Andrei Thomas-Tikhonenko' \\ ${ }^{1}$ Department of Pathobiology and ${ }^{2}$ Department of Animal Biology, School of Veterinary Medicine, \\ 3Department of Pathology and Laboratory Medicine, School of Medicine, and ${ }^{4}$ Biomedical Informatics Core, University of Pennsylvania, \\ Philadelphia, Pennsylvania, USA. ${ }^{5}$ The Wistar Institute, Philadelphia, Pennsylvania, USA. ${ }^{6}$ Leukaemia Research Fund Immunodiagnostics Unit, \\ Nuffield Department of Clinical Laboratory Sciences, John Radcliffe Hospital, Oxford, United Kingdom.
}

\begin{abstract}
The presumed involvement of paired box gene 5 (PAX5) in B-lymphomagenesis is based largely on the discovery of Pax 5 -specific translocations and somatic hypermutations in non-Hodgkin lymphomas. Yet mechanistically, the contribution of Pax 5 to neoplastic growth remains undeciphered. Here we used 2 Myc-induced mouse B lymphoma cell lines, Myc5-M5 and Myc5-M12, which spontaneously silence Pax5. Reconstitution of these cells with Pax5-tamoxifen receptor fusion protein (Pax5ER ${ }^{\mathrm{TAM}}$ ) increased neoplastic growth in a hormone-dependent manner. Conversely, expression of dominant-negative Pax 5 in murine lymphomas and PAX5 knockdown in human lymphomas negatively affected cell expansion. Expression profiling revealed that Pax 5 was required to maintain mRNA levels of several crucial components of $B$ cell receptor (BCR) signaling, including CD79a, a protein with the immunoreceptor tyrosine-based activation motif (ITAM). In contrast, expression of 2 known ITAM antagonists, CD22 and PIR-B, was suppressed. The key role of BCR/ITAM signaling in Pax5-dependent lymphomagenesis was corroborated in Syk, an ITAM-associated tyrosine kinase. Moreover, we observed consistent expression of phosphorylated BLNK, an activated BCR adaptor protein, in human B cell lymphomas. Thus, stimulation of neoplastic growth by Pax 5 occurs through BCR and is sensitive to genetic and pharmacological inhibitors of this pathway.
\end{abstract}

\section{Introduction}

Both normal hematopoiesis and lymphomagenesis are driven, to a large extent, by expression of lineage-specific transcription factors. Many such factors are overtly oncogenic. For example, the hallmark of Burkitt lymphoma and some diffuse large B cell lymphomas (DLBCLs) is the $t(8 ; 14)$ translocation that places the MYC protooncogene under control of the Ig heavy chain gene enhancer $(1,2)$; a similar translocation has been identified in murine plasmacytomas (3). The causative role of Myc in $\mathrm{B}$-lymphomagenesis has been validated through the generation of various transgenic (4-7) and nontransgenic (8) murine models of non-Hodgkin lymphoma.

Other transcription factors are not known to initiate B-lymphomagenesis, but are consistently mutated in full-fledged neoplasms. One such intriguing protein is paired box gene 5 ( $\mathrm{Pax} 5)$, a B cell activator protein (9) with a characteristic paired box DNA-binding motif (10). Inactivation of Pax5 via homologous recombination precludes normal B cell development $(11,12)$, and several lines of evidence implicate Pax 5 as an important factor in B-lymphomagenesis as well.

Nonstandard abbreviations used: BCR, B cell receptor; DLBCL, diffuse large B cell lymphoma; ITAM, immunoreceptor tyrosine-based activation motif; 4OHT, 4 hydroxytamoxifen; Pax5ER ${ }^{\text {TAM }}$, fusion of Pax5 and tamoxifen receptor; shRNA, short hairpin RNA.

Conflict of interest: The authors have declared that no conflict of interest exists. Citation for this article: J. Clin. Invest. 117:2602-2610 (2007). doi:10.1172/JCI30842.
Pax 5 and Myc were the only 2 transcription factors consistently overexpressed in human follicular lymphoma cells compared with their putative normal counterparts, germinal center B cells (13). Similarly, in DLBCLs, MYC and PAX5 were the only 2 transcription factor-encoding genes with high frequencies of hypermutations $(14,15)$. Mutations in Pax5 were also found, albeit with lower frequency, in Burkitt lymphomas with translocated Myc (16). Importantly, PAX5 mutations cluster in regulatory sequences surrounding exon $1 \mathrm{~B}$, suggestive of its overexpression. Overall, high levels of Pax 5 have been reported in almost all non-Hodgkin lymphomas, but in very few acute leukemias (17). Genetic data also support the involvement of Pax5 in lymphomagenesis. A relatively rare (18) but persistent $t(9 ; 14)(\mathrm{p} 13 ; \mathrm{q} 32)$ translocation in various non-Hodgkin lymphomas (19) involves PAX5 (20-22). Furthermore, in acute lymphoblastic leukemia, PAX5 is fused to the ETV6/TEL gene (23).

Yet, despite the large body of circumstantial evidence, the role of Pax5 in B-lymphomagenesis has not been experimentally verified. Pax5 levels have been shown to correlate with (24) or directly promote (25) neoplastic growth in tumors of neural origin (astrocytoma and neuroblastoma, respectively). Curiously, reconstruction of the $\mathrm{t}(9 ; 14)(\mathrm{p} 13 ; \mathrm{q} 32)$ translocation in the knock-in mouse resulted in T-lymphomas, not B-lymphomas (26). To complicate the matter, recently identified translocations in B cell acute lymphoblastic leukemia involving PAX5 result in the creation of its dominant-negative or loss-of-function versions, rather than gainof-function mutants $(27,28)$. While at the molecular level, Pax5 
A

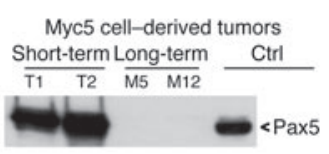

B

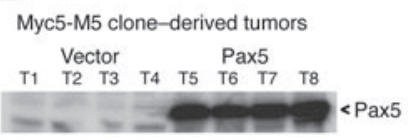

E

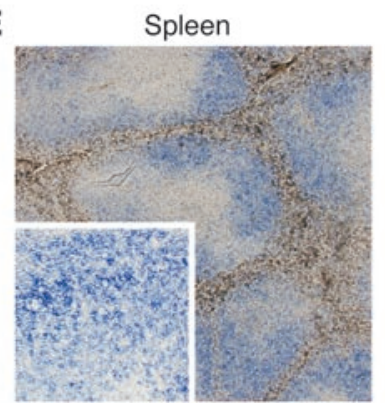

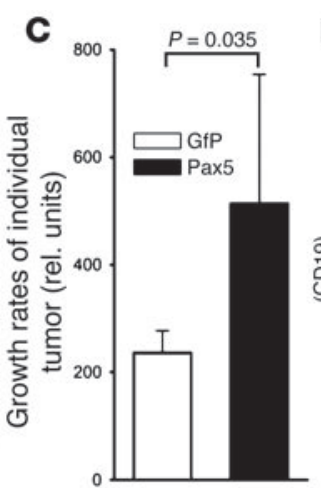

Control tumor

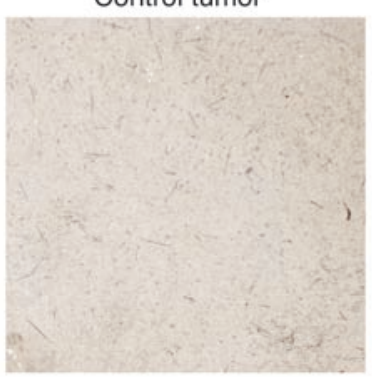

D

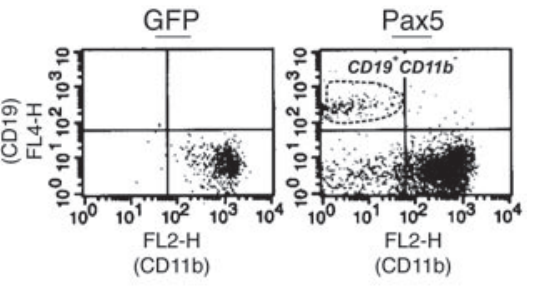

Pax5 tumor

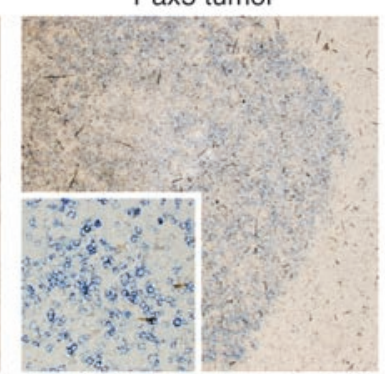

Figure 1

Contribution of Pax5 to neoplastic growth. (A) Immunoblotting demonstrating reactivation of Pax5 in 2 independent Myc5 tumors obtained from short-term cultures (T1 and T2) and lack of such reactivation in Myc5 tumors derived from long-term cultures (M5 and M12). Myc5 cultures engineered to overexpress Pax5 (Ctrl) were used as a positive control. (B) Myc5-M5 cells transduced with the Pax5 retrovirus maintain Pax5 expression in vivo (T5-T8). Vector-transduced cells were used as a negative control (T1-T4). (C) Pax5-transduced Myc5-M5 cells form more rapidly growing tumors than vector-transduced cells. Plotted on the $y$ axis are average slope values. Error bars denote SD. In this and subsequent tumor load experiments, no less than 7 mice were used for each treatment group. (D) Flow cytometric analysis of representative tumors from C. Control tumors (left) were positive for the myeloid marker CD11b and negative for the B cell marker CD19. Pax5-transduced tumors (right) contain both CD11b-CD19- and CD11b-CD19+ cells. (E) Staining of formalin-fixed, paraffin-embedded sections with an antibody against $\mathrm{B}$ cell marker CD45R. Vector-transduced control tumors did not contain CD45R ${ }^{+}$cells. Some Pax5-transduced tumors contain islets of CD45R ${ }^{+}$ cells (blue stain). Normal spleen was used as a positive control. Original magnification, $\times 4$ (insets, $\times 20$ ).

has been reported to transactivate the Epstein-Barr virus promoter (29) and repress expression of p53 (30), these events have not been validated in tumor contexts.

To directly address the role of PAX5 in B-lymphomagenesis, we took advantage of the previously derived series of Myc-induced murine B-lymphomas that spontaneously silence Pax5 upon culturing in vitro (31-34). We used these cells to determine the molecular and cellular consequences of forced Pax 5 reexpression in the context of hematopoietic neoplasms. Our data indicate that Pax5 promotes B-lymphomagenesis via activation of ligand-independent (tonic) B cell receptor (BCR) signaling (35) and that Pax5dependent neoplastic growth is sensitive to genetic and pharmacological inhibitors of this pathway.

\section{Results}

Ectopic expression of Pax 5 promotes tumor growth. Myc5 cells spontaneously downregulate Pax5 after short-term culturing in vitro (31-34). Nevertheless, neoplasms derived from short-term cultures rapidly reactivated Pax5 (Figure $1 \mathrm{~A})$. In contrast, many longterm cultures lost the ability to reexpress Pax5 (Figure 1A). When such cultures, referred to herein as Myc5-M5 and Myc-M12, were transduced with the retrovirus encoding murine Pax5, they produced robust amounts of Pax5 in vivo, while no Pax5 expression was detected in vector-transduced Myc5-M5 cells (Figure 1B and data not shown). When tumor growth rates were compared, Pax5derived neoplasms increased in volume at almost 3 times the rate of control tumors (Figure 1C).

Pax5-transduced tumors were characterized with respect to $B$ cell and macrophage markers (CD19/CD45R and CD11b, respectively). While control neoplasms were CD19-CD11b+, consistent with their macrophage-like phenotype (31-34), expression of Pax5 resulted in the emergence of both double-negative and canonical CD $19^{+}$CD $11 b^{-}$populations (Figure 1D). To corroborate partial reversion to $\mathrm{B}$ cell phenotype, we stained formalin-fixed, paraffin-embedded tumors for CD45R, another B cell marker. In some but not all tumors, we observed isolated clusters of CD45R ${ }^{+}$cells (Figure 1E, right). CD45R $\mathrm{R}^{+}$cells were never seen in Pax5-negative control tumors (Figure 1E, middle). We thus concluded that Pax5 enhances tumor growth while promoting B cell differentiation.

To corroborate this conclusion, we also generated Myc5-M5 and Myc-M12 cells expressing the fusion of Pax5 and tamoxifen receptor $\left(\mathrm{Pax} 5 \mathrm{ER}^{\mathrm{TAM}}\right)$, whose function is completely dependent on 4-hydroxytamoxifen (4OHT) (36). Pax5ERTAM expression levels were comparable to those achieved in Myc5-M5/Pax5 cells with or without hormone treatment (Figure 2A and data not shown). However, the nuclear localization of Pax5ER ${ }^{\text {TAM }}$ was markedly increased in the presence of $4 \mathrm{OHT}$, as evidenced by immunocytochemical staining (Figure 2B). 
A

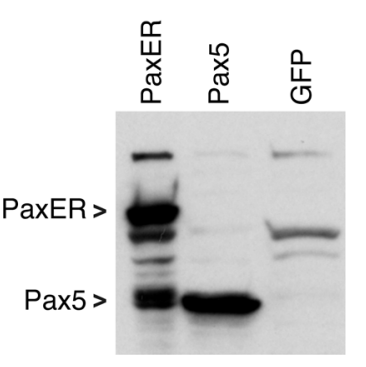

B
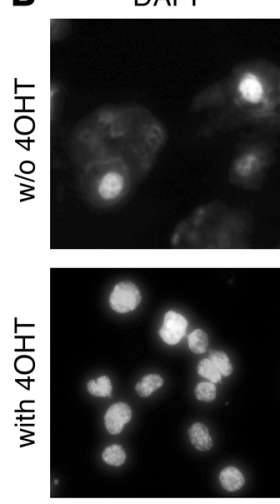

Anti-Pax5 Ab

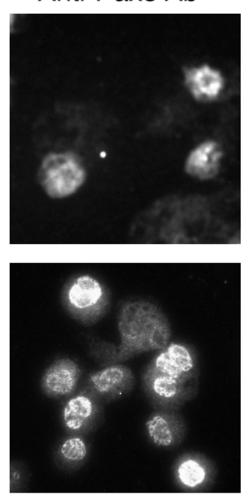

D

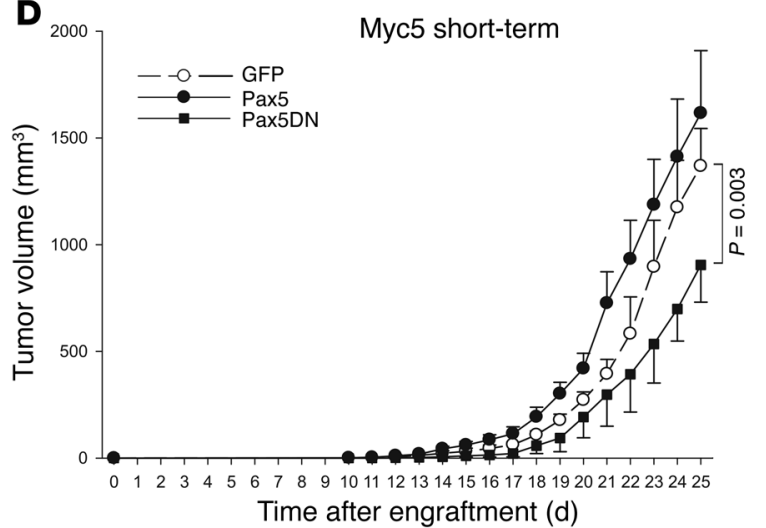

C

Myc5-M5
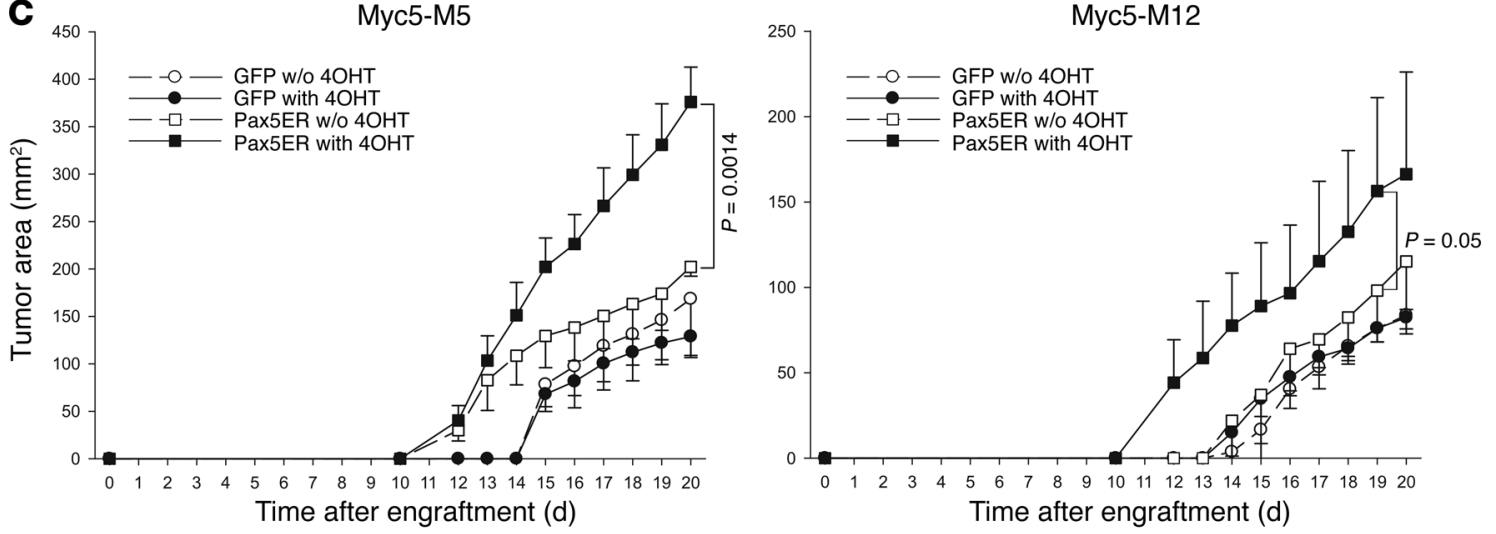

Figure 2

Growth of tumors with conditionally active Pax5. (A) Reconstitution of Myc5-M5 cells with Pax5ER. Immunoblotting on transduced cell lysates was performed using an anti-Pax5 antibody. (B) Enhanced nuclear localization of Pax5ER following treatment with 4OHT. Right panels show immunocytochemical staining with an anti-Pax5 antibody. Left panels show counterstaining of nuclei with DAPI. (C) Kinetics of tumor growth by Pax5ER and control (GFP only) cells in animals treated with 4OHT or vehicle only. (D) Kinetics of tumor growth by shortterm Myc5 cultures expressing either Pax5 or its dominant-negative mutant (Pax5DN) lacking the activation domain. The small increase in tumor size conferred by Pax5 expression was not statistically significant; the decrease in tumor sizes conferred by overexpression of the dominant-negative Pax5 mutant was significant.

To assess the contribution of Pax5ER ${ }^{\mathrm{TAM}}$ to tumor growth in vivo, we injected Myc5-M5/Pax5ER ${ }^{\text {TAM }}$ and Myc5-M12/Pax5ER ${ }^{\text {TAM }}$ cells into mice and randomized the recipients into 2 groups. Half of the mice received daily injections of 4OHT, and the other half was treated with vehicle only. As evidenced by data in Figure 2C, both Myc5-M5- and Myc5-M12-derived Pax5ERTAM cells formed much larger tumors in 4OHT-treated mice. No effect of $4 \mathrm{OHT}$ on growth of control cells was observed.

To demonstrate that the effects of Pax5 are not limited to Myc5derived single cell clones, we also transduced short-term Myc5 cultures with wild-type Pax5 and its dominant-negative mutant (37). Retrovirally encoded Pax5 had a minimal effect on tumor growth, consistent with high levels of the endogenous protein in these tumors (Figure 2D). In contrast, dominant-negative Pax5 significantly inhibited neoplastic growth, attesting to the role of Pax5 in B-lymphomagenesis.

Pax 5 maintains expression of multiple components of BCR signaling. To reveal the mechanism of Pax5-dependent lymphomagenesis, we performed microarray analysis of gene expression in 4OHTtreated GFP-only and Pax5ER ${ }^{\mathrm{TAM}}$ tumors and found that 280 genes were activated more than 2.5-fold in Pax5ERTAM tumors. The list of Pax5-activated genes was run against the KEGG
Pathway database (38), as implemented by DAVID version 2.1 software (39). The strongest similarity $(P<0.005)$ emerged with the components of BCR signaling: 6 out of 280 genes belonged to that pathway. Activation of Pax5ER ${ }^{\mathrm{TAM}}$ caused concerted upregulation of CD79a and CD19 antigens, B cell linker (Blnk), Bruton agammaglobulinemia tyrosine kinase (Btk), PKC $\beta 1$, and phospholipase $\mathrm{C} \gamma$ (Table 1). All these proteins are crucial components of BCR signaling (Figure 3A), and while some of them are already known to be regulated by Pax5 (40-42), the connections between Pax 5 and Btk, PKC $\beta 1$, and PLC $\gamma$ have not yet been reported. Additionally, the analysis of Pax $5 \mathrm{ER}^{\mathrm{TAM}}$-repressed genes revealed downregulation of 2 crucial inhibitors of $B C R$ signaling: CD22 and paired-Ig-like receptor B (PIR-B) (Figure 3A). For all these genes, microarray data were validated using a combination of immunoblotting (Figure 3B), flow cytometry (Figure 3C), and quantitative RT-PCR (Figure 3D). We thus concluded that Pax5 is required to maintain $\mathrm{BCR}$ signaling in vivo.

$B C R$ signaling mediates Pax5-dependent lymphomagenesis. To assess the significance of BCR signaling for Pax5-enhanced neoplastic growth, 3 approaches were taken. In the first set of experiments, we recapitulated BCR signaling using MAHB, a fusion protein containing the cytoplasmic regions of BCR $\operatorname{Ig} \alpha$ and $\operatorname{Ig} \beta$. Target- 
Table 1

Individual BCR signaling components activated by Pax5

\begin{tabular}{lccc} 
Affymetrix ID & Gene & Species & Fold change \\
1426926_at & Plcg2 & Mus musculus & 2.7 \\
1451780_at & Blnk & Mus musculus & 20 \\
1418830_at & Cd79a & Mus musculus & 324 \\
1422755_at & Btk & Mus musculus & 2.6 \\
1423478_at, 1460419_a_at & Prkcb1 & Mus musculus & 2.6 \\
1450570_a_at & Cd19 & Mus musculus & 148 \\
\hline
\end{tabular}

Fold change refers to the ratio of steady-state mRNA levels in Pax5-sufficient versus -deficient cells.

(Figure 4F). Thus, pharmacological inhibition of Pax 5 can be mimicked by inhibiting a BCR/ITAM-associated tyrosine kinase.

Pax 5 and CD22 affect cell accumulation in a BCR ligand-independent manner. To test whether Pax5-induced BCR signaling is ligand-dependent or constitutive (tonic), we measured the effects of Pax5 and CD22 on cell accumulation in vitro, in the absence of any putative BCR ligands. We observed that activation of Pax5ERTAM with

ing of MAHB to the plasma membrane was achieved through the use of myristoylation/palmitoylation signal of Lck. Previous studies have documented the ability of MAHB to trigger constitutive or tonic signals similar to those generated by the BCR in the absence of antigen $(43,44)$. In addition, we used a signaling-deficient MAHB mutant (MAHBmut) variant, in which the crucial immunoreceptor tyrosine-based activation motif (ITAM) tyrosines (Y182F and Y193F in Ig $\alpha$ and $\mathrm{Y} 195 \mathrm{~F}$ and $\mathrm{Y} 206 \mathrm{~F}$ in $\operatorname{Ig} \beta$ ) are substituted with phenylalanines.

Transduction of Myc5-M5 cells with the active MAHB-encoding retrovirus resulted in strong stimulation of tumor growth. ITAM-expressing tumors grew at a rate similar to that of Pax5expressing tumors and much faster than did control GFP-only and MAHBmut neoplasms (Figure 4A). Resultant tumors maintained expression of retrovirally encoded MAHBs and Pax5, as evidenced by immunoblotting (Figure 4B). Thus, MAHB mimics the effects of Pax5 on lymphomagenesis.

In the second set of experiments, we engineered Myc5-M5/ Pax5ER ${ }^{\text {TAM }}$ cells to overexpress CD22, which antagonizes ITAM activity, mainly through the recruitment of Shp1 phosphatase (Figure $3 \mathrm{~A}$ and ref. 45 ). In vector-transduced Pax5ERTAM cells, almost no CD22 was detected. However, transduction with a CD22-encoding retrovirus resulted in readily detectable levels of this protein (Figure 4C). Next, Myc5-M5/Pax5ERTAM/CD22 and Myc5-M5/Pax5ERTAM/puro cells were used for tumor production in vehicle-treated and 4OHT-treated mice. In the absence of active Pax5ER ${ }^{\text {TAM }}$ and without 4OHT, CD22 inhibited tumor growth rather weakly, consistent with the inactive BCR status of these cells. In contrast, tumor sizes in 4OHT-treated animals in which Pax5ER ${ }^{\mathrm{TAM}}$ was active were reduced more than 3-fold (Figure 4D). In fact, in the presence of CD22, there was no difference in growth rates between 4 OHT-treated and untreated tumors. Thus, inhibition of BCR pathway by CD22 completely cancels the effects of Pax5 on tumor growth.

In the third set of experiments, we asked whether pharmacological inhibition of Pax5ERTAM and BCR signaling in preexisting tumors negatively affects neoplastic growth. To this end, Myc5-M5/Pax5ER ${ }^{\mathrm{TAM}}$ tumors were allowed to form in mice continuously treated with 4OHT, after which 4 OHT administration was discontinued in half of the animals. This quickly resulted in tumor stasis: 24 hours after randomization, there was a statistically significant difference in tumor size between treated and untreated animals (Figure 4E). Next, the effects of $4 \mathrm{OHT}$ withdrawal were compared with the effects of Syk inhibitor piceatannol, because the Syk kinase is recruited to all ITAM complexes. We observed that $4 \mathrm{OHT}$ withdrawal and piceatannol treatment inhibited tumor growth with very similar kinetics
4OHT increased cell accumulation (Figure 5A). This was most likely the result of increased cell proliferation, as ${ }^{3} \mathrm{H}$-thymidine incorporation was also increased. No effects of $4 \mathrm{OHT}$ on proliferation of GFP-only cells were apparent (Figure 5A). Moreover, when CD22 was introduced into Pax5ER TAM cells, it had no effect in the absence of 4OHT, but strongly inhibited cell accumulation in cells with active Pax5ER ${ }^{\text {TAM }}$ (Figure 5B). These effects closely resembled the in vivo effects of CD22 (Figure 4D), suggesting that CD22 inhibits ligand-independent BCR signaling brought about by activation of Pax 5 .

PAX5 contributes to neoplastic growth in human B cell neoplasms. To determine whether our findings apply to spontaneous human lymphomas, we first knocked down PAX5 expression in DLBCL lines previously reported to contain activated BCR (46). We transduced SUDHL-4 and SUDHL- 6 with a series of lentivirally encoded short hairpin RNAs (shRNAs) and monitored PAX5 expression using immunoblotting (Figure 5C). Cells transduced with the most efficient hairpin, 6061, were chosen for further analysis. When 48-hour cell accumulation assays were performed, we observed that the 6061 shRNA significantly decreased growth of both DLBCL cultures (Figure 5D), attesting to growth-promoting effects of PAX5 in human cells.

To further corroborate this notion, we stained tissue arrays of human DLCBLs for both PAX5 and phosphorylated BLNK, the principal adaptor protein of activated BCR. Multiple myeloma samples, which are known to silence PAX5 $(47,48)$, were used as a negative control. We observed that all DCBCL tumors stained positively for PAX5, and approximately half of them were also positive for phosphorylated BLNK (Figure 6; both positive and negative samples are shown). Thus, BCR is likely to be constitutively activated in at least some human B cell tumors, attesting to the important causative role in PAX5 for B-lymphomagenesis.

\section{Discussion}

BCR signaling regulates $\mathrm{B}$ cell fate decisions during normal development $(49,50)$, primarily through $\operatorname{Ig} \alpha(C D 79 a)$ and $\operatorname{Ig} \beta$ (CD79b) molecules that contain ITAMs in their cytoplasmic domains (35). Precursors with a functional BCR can differentiate into naive $\mathrm{B}$ cells and leave the bone marrow, and those without undergo apoptosis (51). Consequently, homozygous deletion of BCR components $(52,53)$ or mutations in the ITAM domains (54) rapidly kill B cells.

It has been proposed that BCR signaling (ligand dependent or independent) can promote survival of neoplastic B-lymphoma cells as well, for instance, via activation of antiapoptotic Mcl1 (55). Increased BCR signaling was indeed observed in cultured B-lymphoid cells (56) and appeared to contribute to their growth 

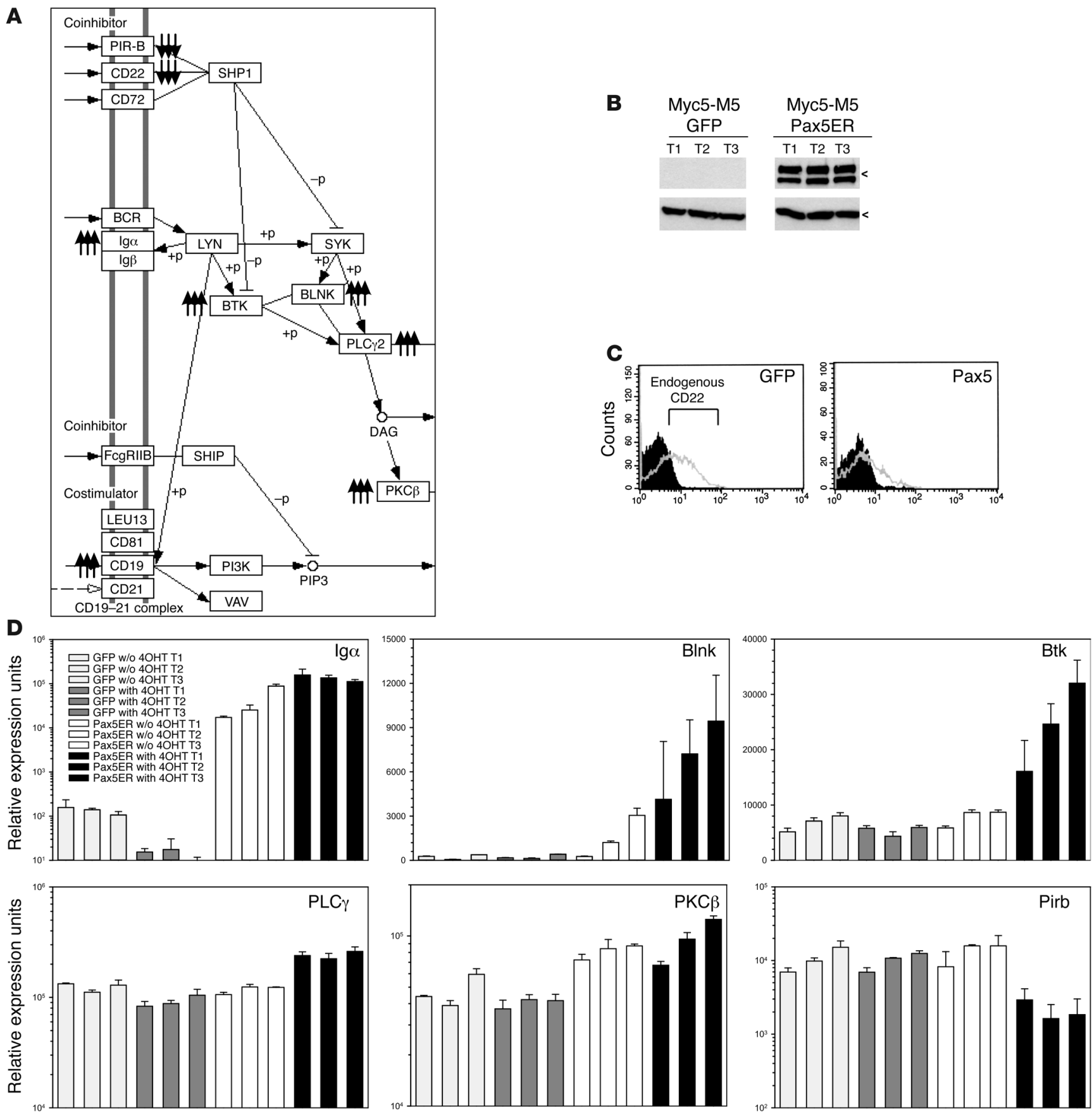

Figure 3

Pax5 affects expression of BCR signaling components. (A) Mapping of Pax5 target genes to the BCR pathway (http://www.genome.jp/dbgetbin/www_bget?path:mmu04662). Triple arrows pointing up and down denote Pax5-activated and -repressed genes, respectively. DAG, diacylglycerol, which is produced by PLC $\gamma 2$ and directly activates PKC $\beta ;+p$, phosphorylation; $-p$, dephosphorylation. (B) Immunoblotting using an anti-CD19 antibody. The 2 bands most likely correspond to the reduced and unreduced forms of the protein (http://mpr.nci.nih.gov/PROW/ guide/1916589419_g.htm). Three independent tumors from each group were tested. All tumors were from 4OHT-treated animals. (C) Flow cytometric detection of CD22 in GFP-only and Pax5-expressing Myc5-M5 tumors. Gray and black plots denote anti-CD22-stained and control cells (not stained with the primary antibody), respectively. (D) Real-time RT-PCR analysis of tumors from Figure 2C. Three independent tumors from each group were used for analysis, and each cDNA was tested in triplicate. Values on the $y$ axis equal $2^{[30-C t]}$. Error bars denote SD. In case of CD79a $(\lg \alpha)$, significant upregulation was observed in Pax5ER ${ }^{\text {TAM }}$ cells even in the absence of $40 H T$. All genes shown are Pax5 with the exception of Pirb, a Pax5-repressed gene. All values are normalized for $\beta$-actin. 
A

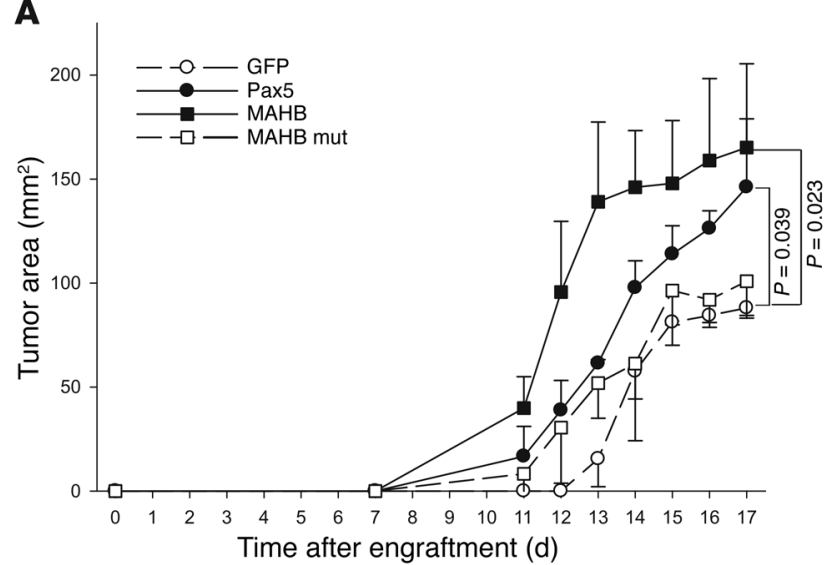

D

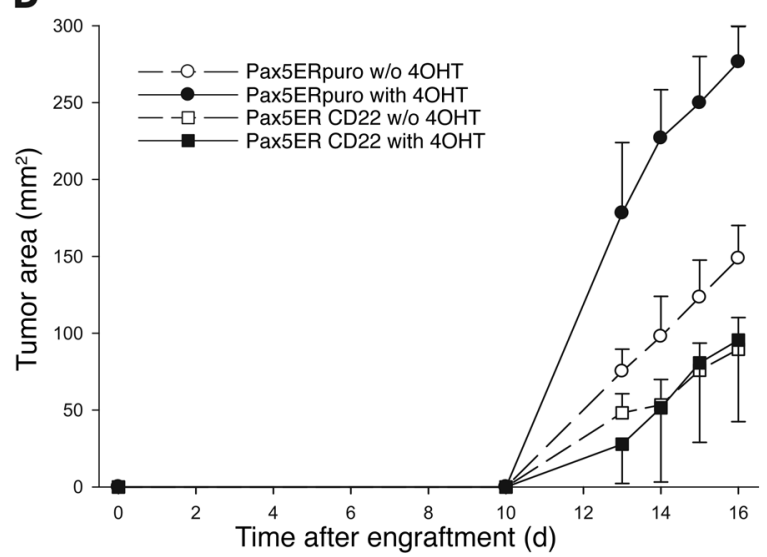

B

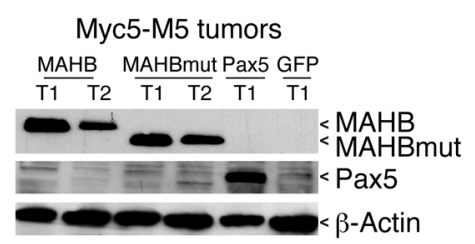

C

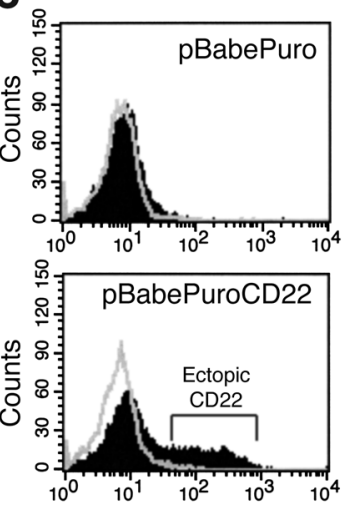

E

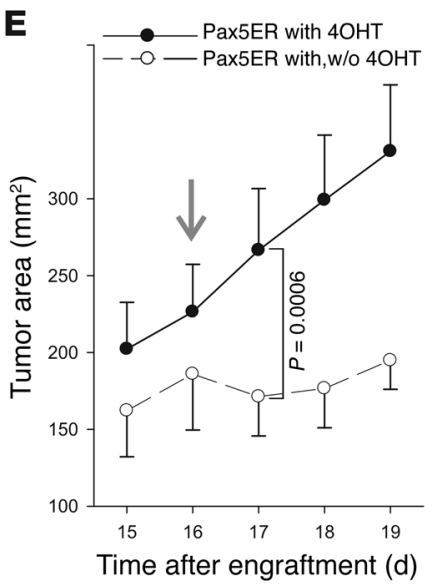

F

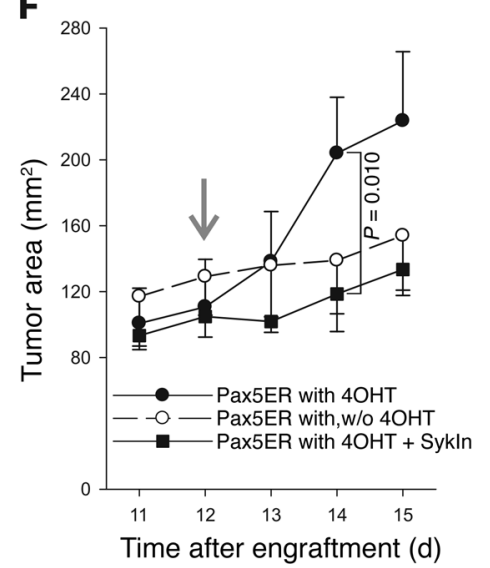

Figure 4

Role of BCR signaling in Pax5-dependent neoplastic growth. (A) Kinetics of tumor growth by Myc5-M5 cells expressing GFP alone, Pax5, MAHB, or the inactive form of MAHB (MAHBmut). Both Pax5 and MAHB significantly promoted tumorigenesis. (B) Immunoblotting detecting ectopic expression of Pax5, MAHB, and MAHBmut in representative tumors from A. (C) Flow cytometric measurement of CD22 levels in Myc5-M5Pax5ER cells transduced by the empty vector or the CD22-encoding retrovirus. (D) Kinetics of tumor growth by cells from $\mathbf{C}$ in animals treated with $4 \mathrm{OHT}$ or vehicle alone. (E) Kinetics of tumor growth by Myc5-M5-Pax5ER cells in animals continuously treated or treated with and subsequently deprived of $4 \mathrm{OHT}$ (with,w/o $4 \mathrm{OHT}$ ). Arrow indicates the day when animals were assigned to different treatment groups. The difference in tumor size between the 2 groups became significant 1 day after randomization. (F) Kinetics of tumor growth by Myc5-M5-Pax5ER cells in animals continuously treated with $4 \mathrm{OHT}$, treated with and subsequently deprived of $4 \mathrm{OHT}$, or treated with $4 \mathrm{OHT}$ and Syk inhibitor (Sykln). Arrow indicates the day when animals were assigned to different treatment groups. The difference in tumor size between the $4 \mathrm{OHT}$ and $4 \mathrm{OHT}$ plus Syk inhibitor groups became significant 2 days after randomization.

(46). Yet the role of BCR signaling in tumor growth in vivo has been inferred only from circumstantial evidence: the persistent expression of BCR in the majority of non-Hodgkin lymphomas, the retention of functional $\mathrm{Ig}$ alleles in lymphomas with $\operatorname{IgH}$ translocations, and the discovery of autoreactive BCR in some neoplasms (reviewed in ref. 57).

Here we demonstrated that the contribution of Pax5 to neoplastic growth correlated with its ability to maintain expression of BCR components. Moreover, it was abolished by interrupting the chain of signaling downstream of CD79a through either overexpression of CD22, which recruits to the complex the Shp1 phosphatase (45), or pharmacological inhibition of Syk, an ITAMassociated tyrosine kinase. Conversely, the constitutively active ITAM construct fully recapitulated the proneoplastic effects of Pax5. These findings not only identify a molecular mechanism whereby Pax 5 promotes neoplastic growth, but directly establish the role of BCR signaling in lymphomagenesis.
It should be noted that some of the newly identified Pax5 targets (e.g., phospholipase $\mathrm{C} \gamma$ and $\mathrm{PKC} \beta$ ) are not restricted to $\mathrm{B}$ cells and participate in a variety of biological processes, such as calcium signaling and NF- $\mathrm{\kappa B}$ activation. It is possible that activation of these and additional genes by Pax 5 contributes to neoplastic transformation of T cell precursors (26).

\section{Methods}

Cell lines and retroviral infection. Generation of Myc5 B-lymphoma cells has been described previously (31). Cells were maintained in RPMI 1640 media supplemented with $10 \%$ fetal bovine serum, IL-7 (0.1 ng/ml; R\&D Systems), and lipopolysaccharide (10 $\mu \mathrm{g} / \mathrm{ml}$; Sigma-Aldrich). Short-term Myc5 cultures were transfected with mouse stem cell virus long terminal repeat-internal ribosome entry site-green fluorescent protein-retrovirus 1-based (MIGR1-based) retroviruses encoding either full-length murine Pax 5 or its dominant-negative mutant, capable of DNA binding but lacking the activation domain (37). Long-term Myc5-M5 and Myc5-M12 cultures 
A

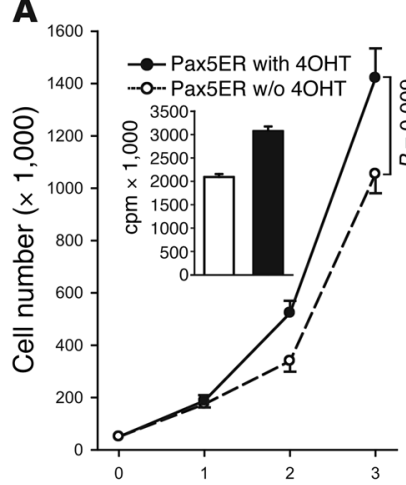

$\mathbf{B}_{5000}$
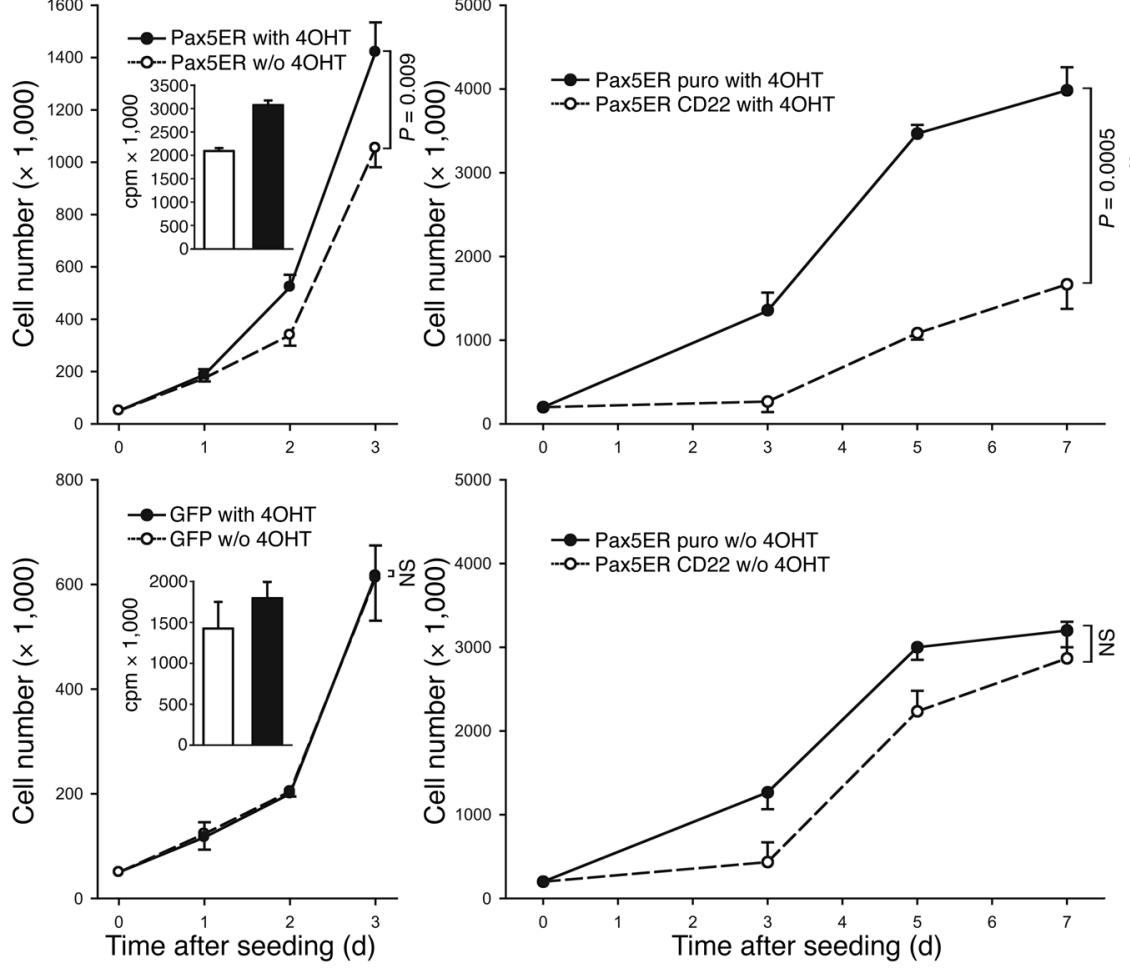

C

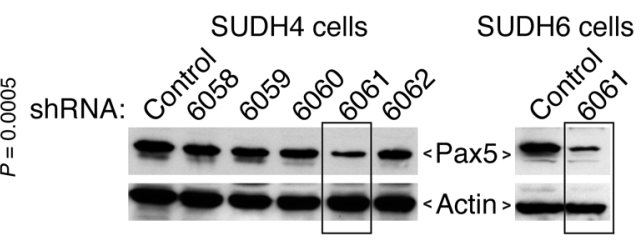

D

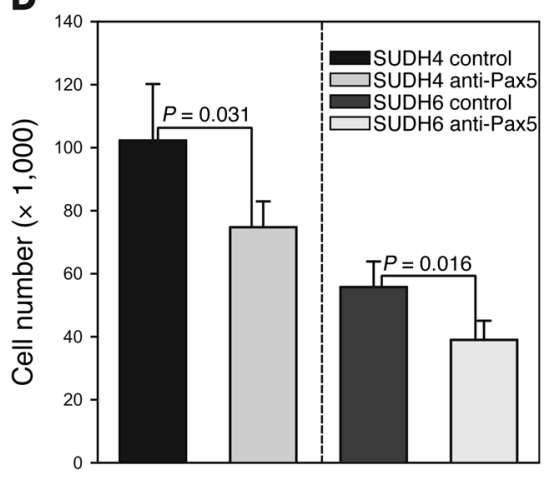

Figure 5

In vitro effects of Pax5 and CD22. (A) Cell accumulation in untreated and 4OHT-treated cultures of Pax5ER and GFP-only Myc5-M5 cells. Cells were counted in triplicate; error bars denote SD. Insets represent ${ }^{3} \mathrm{H}$-thymidine incorporation assay performed on the same cultures. (B) Cell accumulation in untreated and 4OHT-treated cultures of CD22- and vector-transduced Myc5-Myc5-Pax5ER cells. (C) Pax5 knockdown in human SUDHL-4 and SUDHL-6 DLBCL lines using lentivirally encoded shRNAs. Five different hairpins were first tested on SUDHL-4 cells. The most effective shRNA, 6061, was also introduced into SUDHL-6 cells. Pax5 and actin levels were determined using immunoblotting. (D) Cell accumulation assay following Pax5 knockdown. Cells transduced by shRNA 6061 and control virus were seeded at equal densities and counted 48 hours later. $P$ values were determined using unpaired Student's $t$ test.

were transfected with retroviruses encoding Pax5, Pax5ER ${ }^{\text {TAM }}$ (36), MAHB, and its mutant variant (44). Myc5-M5/Pax5ER ${ }^{\mathrm{TAM}}$ cells were also superinfected with pBabePuro CD22 retrovirus. All retroviruses were generated using transfection with Lipofectamine 2000 (Invitrogen) into the GP293 packaging cells. Infections were carried out over the course of 36 hours in the presence of polybrene $(4 \mu \mathrm{g} / \mathrm{ml})$. Human DLBCL lines SUDHL-4 and SUDHL-6 were cultured as described previously (58).

Proliferation assays and cell counts. Cells were seeded in 6-well plates $\left(5 \times 10^{4}\right.$ cells per well) and cultured in the presence or absence of $4 \mathrm{OHT}$. For each condition, the experiment was performed in triplicate. For ${ }^{3} \mathrm{H}$-thymidine incorporation assay, the radioactive compound $[3 \mathrm{H}]$ was added $(1 \mu \mathrm{Ci} / \mathrm{ml})$ 24 hours prior to cell harvest. DNAs were prepared using a PHD Cell Harvester (Cambridge Technology), and incorporated isotope was quantitated using liquid scintillation.

Flow cytometry and immunocytochemistry. Analysis of GFP expression was performed by flow cytometry on FACSCalibur (BD), and the results were analyzed using CellQuest software (BD). The allophycocyanin-conjugated anti-mouse CD19, CD11b, and CD22 were obtained from eBiosciences. Cell sorting for $\mathrm{CD} 22^{\text {high }}$ and $\mathrm{CD} 22^{\text {low }}$ was performed using a FACStar Plus flow cytometer (BD). For immunocytochemistry, cells were spun onto slides, air-dried for 5 minutes, fixed, and stained first with a polyclonal anti-Pax 5 antibody and then with FluoroLink Cy3-labeled goat anti-rabbit IgG (Amersham Biosciences). Images were captured and analyzed using a Zeiss fluorescence microscope.
Tumor load studies. All animal experiments have been reviewed and approved by the University of Pennsylvania Institutional Animal Care and Use Committee. For in vivo experiments, $10^{7}$ Myc5-M5 cells transduced with various constructs were injected subcutaneously into SCID mice (National Cancer Institute). For 4OHT treatment, the hormone powder (H6278; Sigma-Aldrich) was dispersed via sonication in corn oil (SigmaAldrich) at a concentration of $10 \mathrm{mg} / \mathrm{ml}$. Where indicated, mice received daily intraperitoneal injections of the drug ( $1 \mathrm{mg} /$ mouse) or vehicle only. Tumor size was measured daily using calipers, and tumor weights were recorded on the day of tumor excision. Syk inhibitor (piceatannol) was obtained from EMD Biosciences and used as a solution in 10\% DMSO. The single doses $(30 \mathrm{mg} / \mathrm{kg})$ were delivered by tail vein injection.

Microarray analyses. Total RNAs from Myc5-M5/MIGR1 and Myc5-M5/ MIGR1/Pax5ER ${ }^{\text {TAM }}$ tumors developed in 4OHT-treated mice were used. cRNAs were synthesized using in vitro transcription with biotinylated CTP and UTP. Labeled cRNAs were hybridized to the Mouse Genome 4302.0 Array chip (Affymetrix) using the standard protocol of Penn Microarray Facility. Affymetrix MAS 5 probeset signals and presence/ absence flags were calculated. The local pooled error test for differential expression as implemented in S+ArrayAnalyzer (version 1.1; Insightful Corp.) was applied with a $1 \%$ Bonferroni multiple testing correction to median interquartile range normalized MAS 5 signal values. The resulting lists were imported into Gene Spring (version 6.1; Silicon Genetics) filtered for Presence (per Affymetrix MAS5 Analysis) in 2 of 2 samples 

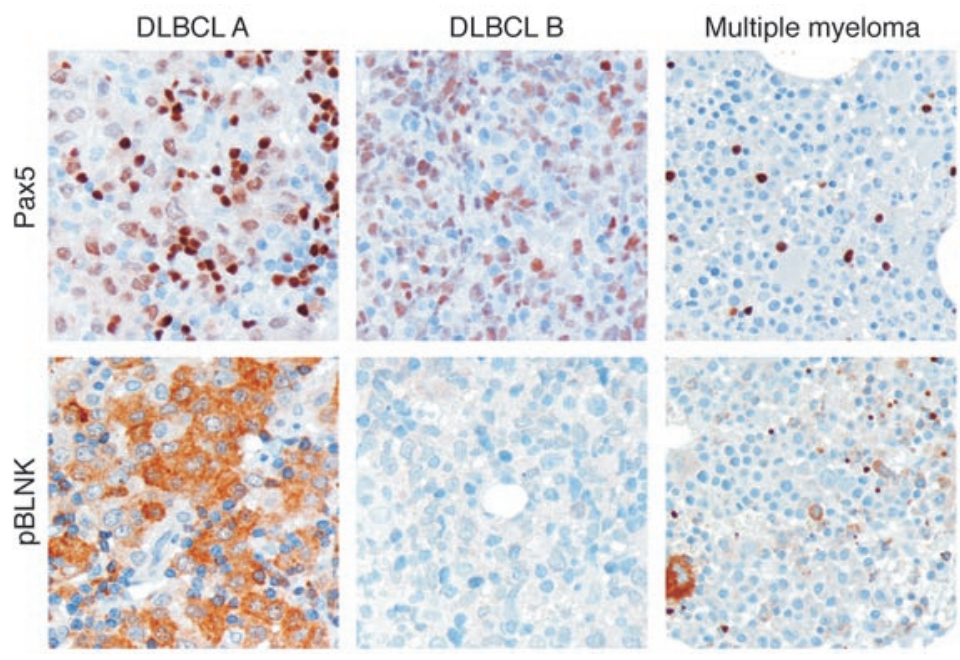

\section{Figure 6}

Immunohistochemical analysis of BLNK phosphorylation in human B cell neoplasms. Archival formalin-fixed, paraffin-embedded sections of 2 diffuse large $B$ cell lymphomas (tumors A and B reflect positive and negative samples, respectively) and 1 multiple myeloma were stained with antibodies against Pax5 and phosphorylated BLNK (pBLNK) as described in Methods. Brown staining indicates positivity for proteins tested. Blue staining depicts counterstained nuclei. Original magnification, $\times 20$.

of 1 or more conditions (GFP or Pax5ERGFP) and then filtered for fold change ( $>2.5$-fold). Primary microarray data have been deposited into the ArrayExpress database (http://www.ebi.ac.uk/arrayexpress) under accession number E-MEXP-1156.

Real-time PCR. Total RNAs were isolated using TRI Reagent (SigmaAldrich) and treated with a TURBO DNA-free kit (Ambion Inc.). cDNAs were prepared from $2 \mu \mathrm{g}$ RNA using SuperScript First-Strand Synthesis System for RT-PCR (Invitrogen). Primers specific for Blnk, Btk, Pirb, Plcg2, Prkcb1, and actin were purchased from QIAGEN. For murine CD79a, the following primers were used: sense, 5'-GCCAGGGGGTCTAGAAGC-3'; antisense, $5^{\prime}$-TCACTTGGCACCCAGTACAA-3'. Amplifications were performed using ABI 7500 Real-Time PCR Machine using Standard7500 SYBR Green real-time protocol. All reactions were performed in triplicate to ensure accuracy of quantitation.

Pax5 knockdown in DLBCL lines. Mission-TRC shRNA-encoding lentiviruses targeting human Pax5 were purchased from Sigma-Aldrich and used for transduction in SUDHL-4 and SUDHL- 6 cell lines according to the manufacturer's protocol. Following transduction, cells were selected in puromycin-containing media $(1 \mu \mathrm{g} / \mathrm{ml})$.

Western blotting. For Pax5 and ITAM expression analysis, cell lysates or tumor lysates were used. CD19 and CD22 expression was detected in tumor lysates. Membranes were probed with the appropriate antibodies diluted according to manufacturers' recommendations. Expression of MAHB and MAHBmut was determined using anti-HA High Affinity antibody (clone 3F10; Roche). Secondary antibodies were used in horseradish peroxidase-conjugated forms (Amersham Biosciences). Antibody binding was detected using the enhanced chemiluminescence system (Amersham Biosciences). Where indicated, a monoclonal antibody reactive with murine actin (Sigma-Aldrich) was used to confirm equal loading.

Immunohistochemistry. Formalin-fixed, paraffin-embedded murine tumors were sectioned and placed on slides, which were then treated with xylene (2 times, 5 minutes each), 4 concentrations of ethanol (100\%, 95\%, 70\% and $50 \%, 1$ minute each), $\mathrm{ddH}_{2} \mathrm{O}$ ( 1 minute), and $1 \times \mathrm{PBS}$ (20 minutes). The sections were blocked for 30 minutes in $1 \times$ PBS, $5 \%$ normal goat serum, and $1 \%$ Tween-20, then stained for 45 minutes with FITC-conjugated B220 antibody (clone RA3-6B2; BD Biosciences - Pharmingen). Slides were washed 3 times in $1 \times$ PBS and $1 \%$ Tween-20, and then stained with mouse FITC-conjugated anti-AP (Sigma-Aldrich). Slides were washed again in $1 \times$ PBS (3 times, 10 minutes each) and developed with Fast Blue BB (SigmaAldrich). All steps were carried out at room temperature. Paraffin-embedded sections from 2 human DLBCL tissue arrays (containing a total of 34 core biopsies) and 4 bone marrow tissue sections with myeloma infiltration were stained essentially as described previously (59). The reagents used were a monoclonal anti-Pax5 (catalog no. 610863; BD Biosciences - Pharmingen) antibody and an antibody against phosphorylated BLNK. Statistics. To determine statistical significance, 2-tailed Student's $t$ test, as implemented by SigmaPlot 9.0 (SYSTAT Software Inc.), was used. Differences with $P$ values of less than 0.05 were considered significant.

\section{Acknowledgments}

We thank Bruce Freedman (University of Pennsylvania) and David Y. Mason (University of Oxford, Oxford, United Kingdom) for stimulating discussions, Hui Wang (University of Pennsylvania) for her help with tail vein injections, Michael Dews for many insightful comments during the course of this project, and Thomas Gilmore (Boston University, Boston, Massachusetts, USA) and Subbarao Bondada (University of Kentucky, Lexington, Kentucky, USA) for DLBCL lines SUDHL-4 and SUDHL-6. This work was supported by NIH grants CA 097932 and CA 102709 to A. Thomas-Tikhonenko and Commonwealth of Pennsylvania Health Research Formula Fund no. 4100020574.

Received for publication November 3, 2006, and accepted in revised form May 29, 2007.

Address correspondence to: Andrei Thomas-Tikhonenko, University of Pennsylvania, M/C 6051, 3800 Spruce Street, Philadelphia, Pennsylvania 19104-6051, USA. Phone: (215) 573-5138; Fax: (215) 746-0380; E-mail: andreit@mail.vet.upenn.edu.

Anna Azvolinsky's present address is: Graduate Program in Molecular Biology, Princeton University, Princeton, New Jersey, USA. 
1. Taub, R., et al. 1982. Translocation of the c-myc gene into the immunoglobulin heavy chain locus in human Burkitt lymphoma and murine plasmacytoma cells. Proc. Natl. Acad. Sci. U. S. A. 79:7837-7841.

2. Dalla-Favera, R., et al. 1982. Human c-myc onc gene is located on the region of chromosome 8 that is translocated in Burkitt lymphoma cells. Proc. Natl. Acad. Sci. U. S. A. 79:7824-7827.

3. Shen-Ong, G.L., Keath, E.J., Piccoli, S.P., and Cole, M.D. 1982. Novel myc oncogene RNA from abortive immunoglobulin-gene recombination in mouse plasmacytomas. Cell. 31:443-452.

4. Adams, J.M., et al. 1985. The c-myc oncogene driven by immunoglobulin enhancers induces lymphoid malignancy in transgenic mice. Nature. 318:533-538.

5. Schmidt, E.V., Pattengale, P.K., Weir, L., and Leder, P. 1988. Transgenic mice bearing the human c-myc gene activated by an immunoglobulin enhancer: a pre-Bcell lymphoma model. Proc. Natl. Acad. Sci. U. S. A. 85:6047-6051.

6. Kovalchuk, A.L., et al. 2000. Burkitt lymphoma in the mouse. J. Exp. Med. 192:1183-1190.

7. Park, S.S., et al. 2005. Insertion of c-Myc into Igh induces B-cell and plasma-cell neoplasms in mice. Cancer Res. 65:1306-1315.

8. Yu, D., and Thomas-Tikhonenko, A. 2002. A nontransgenic mouse model for B-cell lymphoma: in vivo infection of $\mathrm{p} 53$-null bone marrow progenitors by a Myc retrovirus is sufficient for tumorigenesis. Oncogene. 21:1922-1927.

9. Hagman, J., and Lukin, K. 2006. Transcription factors drive B cell development. Curr. Opin. Immunol. 18:127-134

10. Barr, F.G. 1997. Chromosomal translocations involving paired box transcription factors in human cancer. Int. J. Biochem. Cell Biol. 29:1449-1461.

11. Urbanek, P., Wang, Z.Q., Fetka, I., Wagner, E.F., and Busslinger, M. 1994. Complete block of early B-cell differentiation and altered patterning of the posterior midbrain in mice lacking Pax5/BSAP. Cell. 79:901-912.

12. Mikkola,I., Heavey, B., Horcher, M., and Busslinger, M. 2002. Reversion of B cell commitment upon loss of Pax5 expression. Science. 297:110-113.

13. Husson, H., et al. 2002. Gene expression profiling of follicular lymphoma and normal germinal center B cells using cDNA arrays. Blood. 99:282-289.

14. Pasqualucci, L., et al. 2001. Hypermutation of multiple proto-oncogenes in B-cell diffuse large-cell lymphomas. Nature. 412:341-346.

15. Montesinos-Rongen, M., Van Roost, D., Schaller, C., Wiestler, O.D., and Deckert, M. 2004. Primary diffuse large B-cell lymphomas of the central nervous system are targeted by aberrant somatic hypermutation. Blood. 103:1869-1875.

16. Gaidano, G., et al. 2003. Aberrant somatic hypermutation in multiple subtypes of AIDS-associated non-Hodgkin lymphoma. Blood. 102:1833-1841.

17. Krenacs, L., et al. 1998. Transcription factor B-cellspecific activator protein (BSAP) is differentially expressed in B cells and in subsets of B-cell lymphomas. Blood 92:1308-1316.

18. Cook, J.R., et al. 2004. Lack of PAX5 rearrangements in lymphoplasmacytic lymphomas: reassessing the reported association with $\mathrm{t}(9 ; 14)$. Hum Pathol. 35:447-454.

19. Offit, K., Parsa, N.Z., Filippa, D., Jhanwar, S.C., and Chaganti, R.S. 1992. t $(9 ; 14)(\mathrm{p} 13 ; \mathrm{q} 32)$ denotes a subset of low-grade non-Hodgkin's lymphoma with plasmacytoid differentiation. Blood. 80:2594-2599.

20. Iida, S., et al. 1996. The $\mathrm{t}(9 ; 14)(\mathrm{p} 13 ; \mathrm{q} 32)$ chromosomal translocation associated with lymphoplas- macytoid lymphoma involves the PAX-5 gene. Blood. 88:4110-4117.

21. Busslinger, M., Klix, N., Pfeffer, P., Graninger, P.G., and Kozmik, Z. 1996. Deregulation of PAX-5 by translocation of the E $\mu$ enhancer of the IgH locus adjacent to two alternative PAX-5 promoters in a diffuse large-cell lymphoma. Proc. Natl. Acad. Sci. U. S. A. 93:6129-6134.

22. Morrison, A.M., et al. 1998. Deregulated PAX-5 transcription from a translocated IgH promoter in marginal zone lymphoma. Blood. 92:3865-3878.

23. Cazzaniga, G., et al. 2001. The paired box domain gene PAX5 is fused to ETV6/TEL in an acute lymphoblastic leukemia case. Cancer Res. 61:4666-4670.

24. Stuart, E.T., Kioussi, C., Aguzzi, A., and Gruss, P. 1995. PAX5 expression correlates with increasing malignancy in human astrocytomas. Clin. Cancer Res. 1:207-214.

25. Baumann Kubetzko, F.B., et al. 2004. The PAX5 oncogene is expressed in $\mathrm{N}$-type neuroblastoma cells and increases tumorigenicity of a S-type cell line. Carcinogenesis. 25:1839-1846.

26. Souabni, A., Jochum, W., and Busslinger, M. 2006. Oncogenic role of Pax5 in the T-lymphoid lineage upon ectopic expression from the immunoglobulin heavy-chain locus. Blood. 109:281-289.

27. Bousquet, M., et al. 2007. A novel PAX5-ELN fusion protein identified in B-cell acute lymphoblastic leukemia acts as a dominant negative on wild-type PAX5. Blood. 109:3417-3423.

28. Mullighan, C.G., et al. 2007. Genome-wide analysis of genetic alterations in acute lymphoblastic leukaemia. Nature. 446:758-764.

29. Tierney, R., Kirby, H., Nagra, J., Rickinson, A., and Bell, A. 2000. The Epstein-Barr virus promoter initiating B-cell transformation is activated by RFX proteins and the B-cell-specific activator protein BSAP/Pax5. J. Virol. 74:10458-10467.

30. Stuart, E.T., Haffner, R., Oren, M., and Gruss, P. 1995. Loss of p53 function through Pax-mediated transcriptional repression. EMBOJ. 14:5638-5645.

31. Yu, D., et al. 2003. Oscillation between B-lymphoid and myeloid lineages in Myc-induced hematopoietic tumors following spontaneous silencing/reactivation of the EBF/Pax5 pathway. Blood. 101:1950-1955.

32. Johnson, K., et al. 2004. B cell-specific loss of histone 3 lysine 9 methylation in the $\mathrm{V}(\mathrm{H})$ locus depends on Pax5. Nat. Immunol. 5:853-861.

33. Hodawadekar, S., et al. 2006. B-lymphoma cells with epigenetic silencing of Pax5 trans-differentiate into macrophages, but not other hematopoietic lineages. Exp. Cell Res. 313:331-340.

34. Hodawadekar, S., Wei, F., Yu, D., Thomas-Tikhonenko, A., and Atchison, M. 2006. Epigenetic histone modifications do not control Igk locus contraction and intranuclear localization in cells with dual B cell-macrophage potential. J. Immunol. 177:6165-6171.

35. Monroe, J.G. 2006. ITAM-mediated tonic signalling through pre-BCR and BCR complexes. Nat. Rev. Immunol. 6:283-294.

36. Chiang, M.Y., and Monroe, J.G. 2001. Role for transcription Pax5A factor in maintaining commitment to the B cell lineage by selective inhibition of granulocyte-macrophage colony-stimulating factor receptor expression. J. Immunol. 166:6091-6098.

37. Maitra, S., and Atchison, M. 2000. BSAP can repress enhancer activity by targeting PU.1 function. Mol. Cell. Biol. 20:1911-1922.

38. Kanehisa, M. 1997. A database for post-genome analysis. Trends Genet. 13:375-376.

39. Dennis, G., Jr., et al. 2003. DAVID: Database for Annotation, Visualization, and Integrated Discovery.
Genome Biol. 4:P3.

40. Schebesta, M., Pfeffer, P.L., and Busslinger, M. 2002. Control of pre-BCR signaling by Pax5dependent activation of the BLNK gene. Immunity. 17:473-485.

41. Fitzsimmons, D., et al. 1996. Pax-5 (BSAP) recruits Ets proto-oncogene family proteins to form functional ternary complexes on a B-cell-specific promoter. Genes Dev. 10:2198-2211.

42. Kozmik, Z., Wang, S., Dorfler, P., Adams, B., and Busslinger, M. 1992. The promoter of the CD19 gene is a target for the B-cell-specific transcription factor BSAP. Mol. Cell. Biol. 12:2662-2672.

43. Bannish, G., Fuentes-Panana, E.M., Cambier, J.C., Pear, W.S., and Monroe, J.G. 2001. Ligand-independent signaling functions for the B lymphocyte antigen receptor and their role in positive selection during B lymphopoiesis. J. Exp. Med. 194:1583-1596.

44. Grande, S.M., et al. 2006. Cellular ITAM-containing proteins are oncoproteins in nonhematopoietic cells. Oncogene. 25:2748-2757.

45. Nitschke, L. 2005. The role of CD22 and other inhibitory co-receptors in B-cell activation. Curr. Opin. Immunol. 17:290-297.

46. Gururajan, M., Jennings, C.D., and Bondada, S. 2006. Cutting edge: constitutive B cell receptor signaling is critical for basal growth of B lymphoma. J. Immunol. 176:5715-5719.

47. Delogu, A., et al. 2006. Gene repression by Pax5 in $\mathrm{B}$ cells is essential for blood cell homeostasis and is reversed in plasma cells. Immunity. 24:269-281.

48. Nera, K.P., et al. 2006. Loss of Pax 5 promotes plasma cell differentiation. Immunity. 24:283-293.

49. Reth, M., and Wienands, J. 1997. Initiation and processing of signals from the B cell antigen receptor. Annu. Rev. Immunol. 15:453-479.

50. Allman, D., Srivastava, B., and Lindsley, R.C. 2004. Alternative routes to maturity: branch points and pathways for generating follicular and marginal zone B cells. Immunol. Rev. 197:147-160.

51. Smith, S.H., and Reth, M. 2004. Perspectives on the nature of BCR-mediated survival signals. Mol. Cell. 14:696-697.

52. Torres, R.M., Flaswinkel, H., Reth, M., and Rajewsky, K. 1996. Aberrant B cell development and immune response in mice with a compromised BCR complex. Science. 272:1804-1808.

53. Lam, K.P., Kuhn, R., and Rajewsky, K. 1997. In vivo ablation of surface immunoglobulin on mature B cells by inducible gene targeting results in rapid cell death. Cell. 90:1073-1083.

54. Kraus, M., Alimzhanov, M.B., Rajewsky, N., and Rajewsky, K. 2004. Survival of resting mature B lymphocytes depends on BCR signaling via the Ig $\alpha / \beta$ heterodimer. Cell. 117:787-800.

55. Petlickovski, A., et al. 2005. Sustained signaling through the B-cell receptor induces $\mathrm{Mcl}-1$ and promotes survival of chronic lymphocytic leukemia B cells. Blood. 105:4820-4827.

56. Irish, J.M., Czerwinski, D.K., Nolan, G.P., and Levy, R. 2006. Altered B cell receptor signaling kinetics distinguish human follicular lymphoma B cells from tumor infiltrating non-malignant B cells. Blood. 108:3135-3142.

57. Kuppers, R. 2005. Mechanisms of B-cell lymphoma pathogenesis. Nat. Rev. Cancer. 5:251-262.

58. Gururajan, M., et al. 2005. c-Jun N-terminal kinase (JNK) is required for survival and proliferation of B-lymphoma cells. Blood. 106:1382-1391.

59. Marafioti, T., et al. 2004. Expression of intracellular signaling molecules in classical and lymphocyte predominance Hodgkin disease. Blood. 103:188-193. 